\title{
Assessment of Adult Nutritional Status and Associated Factors Among ART Users in Nekemte Referral Hospital and Health Center, East Wollega Zone, Ethiopia
}

\author{
Meskerem Alemayehu Kenea ${ }^{1}$, Sileshi Garoma ${ }^{2}$, Habtamu Fekadu Gemede ${ }^{2,3}$, * \\ ${ }^{1}$ Gatema Secondary High School, Nekemte, Ethiopia \\ ${ }^{2}$ Nutrition and Food Science Program, Wollega University, Nekemte, Ethiopia \\ ${ }^{3}$ Center for Food Science and Nutrition, Addis Ababa University, Nekemte, Ethiopia \\ Email address: \\ fekadu_habtamu@yahoo.com (H. F. Gemede)
}

\section{To cite this article:}

Meskerem Alemayehu Kenea, Sileshi Garoma, Habtamu Fekadu Gemede. Assessment of Adult Nutritional Status and Associated Factors Among ART Users in Nekemte Referral Hospital and Health Center, East Wollega Zone, Ethiopia. Journal of Food and Nutrition Sciences. Vol. 3, No. 2, 2015, pp. 56-63. doi: 10.11648/j.jfns.20150302.15

\begin{abstract}
The aim of this study was to assess nutritional status and associated risk factors of patients living with HIV/AIDS following Anti Retro viral Therapy at Nekemte Referral Hospital and Nekemte health center, Ethiopia. A cross-sectional descriptive study was conducted from January 15, 2013 to March, 2013 in Nekemte Referral Hospital and health center. A total of 423 ART users are selected by systematic random sampling technique from all adult ART clients will be included in the study area. The data will be collected by one physician, three nurses, two Health Officers and three data clerks. Clinical assessments, measurements of height and weight and structured questionnaire based interview will be used for data collection after the proper consent and ethical clearance. In this study the prevalence of malnutrition in HIV/AIDS clients who attend ART was $(27 \%)$. Both HIV/AIDS and malnutrition independently cause progressive damage to the immune system and increased susceptibility to infection. ART interruption and presence of opportunistic infection (tuberculosis) were significantly associated with nutritional statuses of the adults on the ART in Nekemte referral hospital and Nekemte health center. In Nekemte Referral Hospital and Nekemte Health center clients are malnourished due to the associated factors such as presence of opportunistic infection that is tuberculosis. From 19 clients that have TB (75.9\%) of them are mal nourished and interrupting treatment was seen to contribute to malnutrition for the respondents. Awareness creation should be made for not to interrupt ART since effective treatment with ART reduces the chance of progression to active TB.
\end{abstract}

Keywords: Adult, Nutritional Status, ART, Nekemte Referral Hospital and Health Center, Associated Factors

\section{Introduction}

As the Human immunodeficiency virus (HIV/AIDS) pandemic continues to expand, the moral imperative to provide safe and efficacious treatment options becomes of paramount interest to the international health-care communities. The use of antiretroviral therapy (ART) has become the cornerstone of the clinical armamentarium available to prevent transmission and slow progression of the infection in people living with HIV/AIDS (PLWHA) worldwide. Efforts have begun for a significant scaling up of the use of antiretroviral drugs (ARVs) in settings such as subSaharan Africa where the epidemic has had its most devastating impact. However, questions have been raised about the use of ART against a background of health problems not often seen in the developed world observed that access to effective therapies for HIV-positive persons is arguably one of the highest global public health priorities but that simply providing affordable access to ARVs is insufficient. Prevention should be combined with access to clinical care that clearly helps patients to live longer, healthier lives (Reynolds et al., 2003)

The World Health Organization (WHO) and UNAIDS estimated that at the end of 2007, there were 33 million people living with HIV infection (PLHIV) worldwide, with $67 \%$ of these living in Sub-Saharan Africa (Aluísio J D Barros, Cesar G Victora, 2005). In Benin it is estimated that 64000 people were living with HIV in 2007. Access to antiretroviral therapy (ART) is increasing in Benin, however coverage is only estimated to be about $50 \%$ or 9800 of 20 
000 PLHIV requiring treatment (Amadi, B. et al. 2001).

Based on the 2007 single point estimate, the Government of Ethiopia (GOE) projected HIV prevalence to be $2.4 \%$ in 2010 with over 1.2 million HIV positive people in the country and a female to male ratio of $1.5: 1.0$. This projected national prevalence characterizes the Ethiopian epidemic as generalized. Notwithstanding, there are wide regional and urban: rural disparities with urban prevalence estimated at $7.7 \%$ or 760,475 people living with HIV/AIDS (PLWHA). This compares starkly with the situation in rural areas, where $85 \%$ of the country's population resides. In this huge territory, twice the size of Texas or France, prevalence is just $0.9 \%(456,432$ PLWHA or $37 \%$ of the national total). Regional prevalence varies substantially ranging from $0.8 \%$ in Somali region to $9.2 \%$ in Addis Ababa. There is also emerging evidence that prevalence amongst some most at risk populations (MARPs) is considerably greater. The GOE also projects that out of 5.4 million orphans in the country, 804,184 (15\%) are due to AIDS (Operational Plan Report 2012).

In Ethiopia, the HIV epidemic has remained a major public health problem, mainly affecting people of prime productive and reproductive age. At the end of 2005, an estimated 1.3 million people were living with HIV ( $\mathrm{MOH}, 2006)$. HIV prevalence in the general population is estimated at 2.1 percent. The epidemic is well established in the urban areas with prevalence as high as 5.5 percent in 2005 . In the same year, 0.7 percent of the rural population was estimated to be HIV positive (CSA, ORC and Macro, 2006).

In a resource poor country, it is currently estimated that 1.5 million people are living with HIV/AIDS. Even though the epidemic is progressing at a slower rate, the rate of change is not sufficient enough to be complacent. It will take a number of years to see noticeable declines in prevalence and incidence reduction with sustained effort, due to the magnitude of the damage already inflicted, and the population size. Despite the odds, the achievements so far are encouraging and Ethiopia has many partners that are working hard to contain the pandemic. As part of this endeavor and as one of its major duties, the government is trying its best to create an enabling policy and intervention environment by putting in place the necessary tools and implementation mechanisms. These include HIV/AIDS policy, the revised strategic framework, and different institutional arrangements for leadership, coordination and monitoring of activities as well as mobilizing resources. The cumulative number of people living with HIV/AIDS is about 1.5 million (3.8\% male and 5\% female; $12.6 \%$ urban \& $2.6 \%$ rural) out of which about 96,000 are children under 15 years. The estimated number of new AIDS in the adult population was 98,000 ( $46 \%$ male \& $54 \%$ female) while that in children was 25,000 . Some 245,000 people living with HIV/AIDS were in need of antiretroviral treatment (ART) in 2003 and some 90,000 adults and 25,000 children had died of AIDS in 2003. Of the 15,580 blood donors from different parts of Ethiopia in 2003, 4.7\% (3.8\% female \& 5.0\% male) were positive for HIV, so were $3.6 \%$ of the 68,273 visa applicants (FMOH 2004).
The government is highly committed to increasing access to antiretroviral therapy. In 2002, Ethiopia approved a national policy providing tax exemption for antiretroviral drugs and related supplies with the objective of providing treatment at a low cost to people in need (WHO 2005).

In January 2005, the government launched a programme to provide access to antiretroviral therapy free of charge and made a commitment to roll out the programme across the country in 2005 and 2006, with the long-term objective of ensuring universal access to treatment. The government also committed to expanding voluntary counseling and testing services across the country along with the roll-out of antiretroviral therapy. Clinical guidelines on the use of antiretroviral drugs have been developed in accordance with international standards. The current model for delivering antiretroviral therapy is physician-led and hospital-based. The health centers provide services for HIV/AIDS chronic care, identify and refer eligible people, follow up people living with HIV/AIDS who are stable and provide information on antiretroviral therapy. The community level provides services for treatment adherence, literacy and preparedness. Antiretroviral drugs are distributed by licensed pharmacists at authorized outlets (WHO 2005).

The expansion of antiretroviral treatment (ART) services has been recent and fast from only four facilities in 2003 to 481 in 2008. The number of people who have accessed ART has increased substantially from 900 in 2003 to 210,637 in 2009. The impact of the program on patient survival and quality of life has also been demonstrated (MOH and HAPCO 2008). A recent population-based study revealed significant decline in adult AIDS mortality as a result of ART scale-up in Addis Ababa (Reniers et al. 2009).

Ethiopia's policy and guidelines on the provision of ART show a strong commitment to equitable access to ART. The documents touch on roles for all levels of the health service provision program, as well as communities and the private sector with the intent that different segments of the population will be served by different sectors all with the end goal of providing an equitable ART program. The country also envisages achieving universal access to ART by the end of 2010 (Road Map II). Thus, equity is certainly among the most important aspects of Ethiopia's policy agenda on ART scale-up. Literature indicates that ensuring a diet with sufficient quantities of nutrient-rich foods is crucial for all people, and nutrition is an integral component of care and support of PLHIV (Tony et al. 2004).

Ethiopia has one of the world's highest incidences of undernourished individuals. Approximately 49\% of the population is without adequate nutrition. About $47 \%$ of children fewer than five experience chronic and $24 \%$ severe malnutrition. Given these high levels of malnutrition and vitamin A deficiency, it is likely that deficiencies in other micronutrients like zinc, iron, folate, and vitamin $\mathrm{C}$ also exist in Ethiopia (CSA 2005 and FAO 2000).

PLHIV are more vulnerable to malnutrition than the general population and nutritional status is a good predictor of their mortality risk. Malnutrition in PLHIV often occurs in 
a background of poverty and lack of access to food. It is not always possible to identify one single cause as the main contributor to declining nutritional status or malnutrition in HIV. PLWHA (People Living With HIV/AIDS) are more likely to become malnourished due to: Reduced food intake resulting from appetite loss and eating difficulty, possibly as a result of side effects of medication, infections, or depression, Poor absorption of nutrients which may be the result of chronic diarrhea and HIV-caused intestinal cell damage, Changes in the way the body uses nutrients it receives or has stored (FDREMOH 2006).

HIV infection and poor nutritional status are interlinked. Measuring the nutritional status of PLWHA is an essential part of ART program. It involves the assessment of risk factors in HIV/AIDS positive persons, by estimating Body Mass Index (BMI) and percent of body weight loss (Judith Nerad et al. 2003; Niyongabo et al. 1997 and Tamsin, A. et al. 2003).

One of the most important observations about the interaction between ART and nutritional status is that initiating ART often leads to a reversal of symptoms caused by HIV such as malnutrition and loss of body mass (including muscle mass). Increased appetite, improved food intake and reduced viral load improve nutritional status. This improvement is associated with a reduction in morbidity and mortality from HIV related causes (Raiten D. et al. 2005). Therefore, the objective of this study is to assess nutritional status and associated factors of adult patients living with HIV/AIDS who are following ART at Nekemte Hospital and Nekemte health center in East Wollega, Ethiopia.

\section{Materials and Methodology}

\subsection{Description of Study Area}

The study was conducted in Nekemte Referral hospital and Nekemte health center ART clinic. This is located in East Wollega Zone at a distance of about $331 \mathrm{kms}$ west of the capital city, Addis Ababa. The population of Nekemte town is about 96,555 with two health centers and one hospital (Nekemte Hospital) serving the population of the town and its surrounding. The ART service in Nekemte health center was initiated in 1999 E.C. while the ART service for Nekemte Referral hospital was initiated in 1998 E.C and has one clinic. This hospital serves people from the zone as well as other neighboring zones: Currently, in the adult ART clinic, there are about 6585 persons on HIV care among which 3544 patients are started and following ART. In Nekemte health center, number of patients enrolled is 1303 among these 605 of them are following ART.

\subsection{Study Design and Period}

Institutional based cross-sectional descriptive study using quantitative method has been used to assess adult nutritional status and associated factors among ART users at Nekemte Referral Hospital and Nekemte health center. The study has been conducted between January and March, 2013.

\subsection{Source Population}

All adult HIV/AIDS patients who are on follow up in Nekemte Referral hospital and Nekemte health center ART clinic.

\subsection{Study Population}

The study population was adult ART clients selected by the systematic sampling method from the source population during study period.

\subsection{Inclusion Criteria}

The inclusion criteria of the study was those who are already started ART and above age of 15 years.

\subsection{Exclusion Criteria}

Patients who have kyphoscoliosis (for height measurement), critically ill and unable to communicate, HIV cases not yet started ART, pregnant woman were excluded from the study.

\subsection{Sample Size Determination}

The sample size of the study was calculated using formula for estimation of single proportion as: (Getu D. and Fassil T., 2002)

$$
n=\frac{\mathrm{z}_{a / 2}^{2} \times p(1-P)}{w^{2}}
$$

Where:

$\mathrm{n}=$ sample size

$\mathrm{p}=$ prevalence of HIV positive individuals with malnutrition, as the proportion is not known from previous studies in the area, it will be taken as $0.5(50 \%)$.

$\mathrm{W}=$ maximum allowable error (margin of error) $=0.05$

$\mathrm{Z}=$ value of standard normal distribution (Z-statistic) at $95 \%$ confidence level $(\mathrm{z}=1.96)$.

By applying the above formula the optimum sample size (n) $=384$ subjects were needed.

The final sample size with $10 \%$ non response rate was 423 patients.

\subsection{Sampling Procedure}

The sampling technique was systematic random sampling by selecting one of the days of the week randomly to start with and selecting the first study subject from registration one to ten randomly and then taking every $10^{\text {th }}$ individual from the list of clients. (Sampling interval is $k=N / n=$ $4149 / 423=10$ ). Where, $\mathrm{N}=$ total clients on ART at Nekemte referral hospital and Nekemte health center, $n=$ number of sample size. Then, this trend was continued from one day to another until the desired sample size was attained data. Data collection was conducted in every working day.

\subsection{Study Variables}

Dependent variable: - nutritional status (BMI) of PLWHA 
on ART.

Independent variables: - Presence/absence of nutritional support, Social variables including economic status, educational level, regimen of ART and stage of HIV disease and presence of opportunistic infection.

\subsection{Data Collection Procedures}

The structured questionnaire which was prepared in English first translated in to Afan Oromo and then translated back to English to check for consistency by doing preliminary test as all the respondents can hear and speak Afan Oromo and pre-tested in Jima Arjo Health center on 5\% of the sample with the data collectors and then modified accordingly. Weighing scale and measuring scale for height was checked to make measurements more reliable. Data on socio-demographic and nutritional history was collected using a structured and pre-tested questionnaire. Clinical staging of the disease was made by physicians using WHO staging criteria (WHO 2005).

\subsection{Nutritional Status (Body Mass Index) Measurement}

\subsubsection{Measurement of Weight}

Measurement on body weight was conducted using a standard beam balance that is used in the medical setup recorded to the nearest $0.1 \mathrm{~kg}$. The body weight was taken with subject light clothed and shoes taken off. Besides, over worn closes such as scarf in case of women were also asked to remove during body weight measurements.

\subsubsection{Measurement of Height}

Similarly height measurements were carried out while the subject removed his/her shoes, stand erect, looking straight in a horizontal plane with feet together and knees straight. During this measurements the heels, buttocks, shoulder blades and the back of the head was adjusted to touch against the wall and the measurements was recorded to the nearest $0.5 \mathrm{~cm}$.

\subsection{Body Mass Index Calculation}

BMI was calculated using the formula: $\mathrm{BMI}=$ Weight in

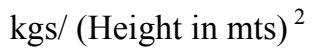

Then classification was made using the standard BMI chart and read as: Take the clients' weight on $\mathrm{X}$ - axis and height on $\mathrm{Y}$-axis. BMI is the point at which the two lines meet.

Data collection was made by two nurses who are assisting in the ART clinic in Nekemte referral hospital and one nurse in Nekemte health center. Training was given to all data collectors on the questionnaire and ways of collecting data by the principal investigator.

\subsection{Data Quality Assurance}

The quality of data was assured through careful design, translation and pretesting of questionnaire, proper training of data collectors and proper handling of data. The data was monitored frequently during collection and collected questionnaires were examined for completeness and consistency during interview and at the end of each day.

\subsection{Data Processing and Statistical Analysis}

Data was checked, coded and entered to SPSS version 20 statistical package for windows. Data entry was conducted by the principal investigator. Prevalence was determined and analysis was made for determination of relationship between associated factors and malnutrition status using Odds ratio and $95 \%$ confidence interval. Multiple regression analysis was used to determine the effect of numerous factors on the outcome variable and to control confounding effect and predict population parameters. All the assumptions of regression analysis (model adequacy and multi-co linearity of independent variables) will be checked to be satisfied using appropriate methods. Cross tabulation were also used to see the association between the explanatory and outcome variables.

\subsection{Ethical Considerations}

Ethical clearance was obtained from Wollega University Ethical Review Committee. And official letter was written to Nekemte Referral hospital and Nekemte health center. The participants were informed that they had the full right to participate or not to participate in the study as well as to with draw any time during the interview. Confidentiality was assured through recoding without a name and coding of questionnaires and placed in safe place after have been collected. Also they were told that any information collected from them would be kept confidential. Consent was obtained from all individual respondents

\section{Result and Discussion}

\subsection{Result}

\subsubsection{Socio-Demographic Characteristics}

A total of 423 adult patients on ART were involved in the study with response rate of $100 \%$ and [59.8\%] of the participants were females and (34.5\%) were males. One hundred fifteen $(27.2 \%)$ were in the age group of $15-29$ years while the majority, (69.3\%) were between 30-49 years and fifteen $(3.5 \%)$ were 50 and above years. About [52.2\%], [10.6\%] and [34.5\%] of respondents were Orthodox, Muslim and Protestant respectively, while the rest were catholic (2.1\%) and Adventist (0.5\%).

Concerning the occupational status of the respondents; (33.6\%), (19.9\%) and (18.9\%) were daily laborer, merchant and government employee except health workers respectively. Regarding the educational status; most of them were attend elementary school $[27.0 \%]$ and secondary school [26.5\%]. (22.5\%) of the respondents could not read and write, whereas $(8.5 \%),(3.5 \%),(9.5 \%)$ and $(2.6 \%)$ of the respondents were read and write only, preparatory, college and university respectively. Regarding marital status more than half of the respondents were married $(54.3 \%)$, while $(27.2 \%)$ were single and $(10.9 \%),(7.6 \%)$ were divorced and widowed respectively. 
Regarding the monthly income of the respondents; majority of them [65.5\%] got $<675 \mathrm{ETB}$ and few of them got $>=675 \mathrm{ETB}$ respectively. The study participants were predominantly Oromo, $(74.5 \%)$ while the rest belongs to other ethnic groups $(25.4 \%)$ (Table 1$)$.

Table 1. Socio-demographic characteristics of adult PLHIV that are on ART, in Nekemte Hospital and Health Center, West Ethiopia, June, 2013.

\begin{tabular}{|c|c|c|c|}
\hline Characteristics [n=423] & Category & Frequency & Percent \\
\hline \multicolumn{4}{|c|}{ Get any nutritional care and support } \\
\hline & Yes & 156 & 36.9 \\
\hline & No & 267 & 63.1 \\
\hline \multirow[t]{2}{*}{ Get dietary counseling } & Yes & 394 & 93.1 \\
\hline & No & 29 & 6.9 \\
\hline \multirow[t]{2}{*}{ Problem with eating } & Yes & 127 & 30 \\
\hline & No & 295 & 69.7 \\
\hline \multirow{3}{*}{ Clinical stage of the disease: } & Stage-II & 103 & 24.3 \\
\hline & Stage-III & 145 & 34.3 \\
\hline & Stage- IV & 5 & 1.2 \\
\hline \multirow[t]{2}{*}{ Treatment interrupted } & Yes & 59 & 13.9 \\
\hline & No & 364 & 86.1 \\
\hline \multirow[t]{4}{*}{ Opportunistic infection } & Chronic diarrhea & 98 & 23.2 \\
\hline & Chronic cough & 56 & 13.2 \\
\hline & Oral \& /or esophageal & 5 & 1.2 \\
\hline & Others & 2 & 0.5 \\
\hline
\end{tabular}

Determinants of Nutritional status and its outcomes

Bivariate logistic regression was used to identify the characteristics that differentiated malnourished study subjects from those well nourished. Coefficients were expressed as crude OR relative to the referent category. However, no statistically significant relationships were observed between sex, age, marital status, educational status, income and nutritional status $(\mathrm{BMI})(\mathrm{P}>0.05)$

\subsubsection{Association of Nutritional Status and Its Associated Factors}

A few of risk factors emerged as significant predictors of nutritional status. Among the predictors opportunistic infection (tuberculosis) and treatment interrupted were significant $(\mathrm{p}<0.05)$. However no statistically significant relationships were observed between ART regimen, nutritional care and support, dietary counseling and clinical stage of the disease and nutritional status $(\mathrm{BMI})(\mathrm{P}>0.05)$

\subsubsection{Association of Different Associated Factors and Nutritional Status}

Different multivariate logistic regression model was employed to evaluate associations between factors and dichotomous variables, designed to measure nutritional status of adult people living with HIV/AIDS that are on ART and coefficients were expressed as Adjusted odds ratio (AOR) relative to reference category and the results of final models were summarized in table 5.Accordingly, nutritional status was found to be significant even after controlling for other factors in the table. Those who reported to have tuberculosis had significantly lower likelihood of malnutrition than other opportunistic infections, OR $(95 \% \mathrm{CI}): 0.30(0.11,0.73)$. In addition respondents interrupted treatment had significantly lower likelihood of malnutrition than those who did not interrupt treatment, OR $(95 \% \mathrm{CI})$ : $0.54(0.30,0.98)$ (Table). The other associated factors are insignificant in bivariate analysis.

Table 2. Association of variables with Nutritional status among the respondents of study, as identified by multivariable analysis, Nekemte Hospital and Health Center, West Ethiopia, March, 2013.

\begin{tabular}{llll}
\hline \multirow{2}{*}{ Variable } & Body Mass Index (BMI) & Malnourished9\%) & Adjusted \\
\cline { 2 - 4 } & Well nourished (\%) & & \\
\hline Treatment interrupted: & & $23(39.0$ & $0.54(0.30,0.98) * *$ \\
Yes & $36(61.0)$ & $89(24.5)$ & 1 \\
No & $274(75.5)$ & & $1.50(0.85,2.66)$ \\
Opportunistic infection: & & $20(20.4)$ & $1.62(0.79,3.34)$ \\
Chronic diarrhea & $78(79.6)$ & $11(19.6)$ & $0.30(0.11,0.73)^{* *}$ \\
Chronic cough & $45(80.4)$ & $11(57.9)$ & $1.65(0.17,15.57)$ \\
Tuberculosis & $8(42.1)$ & $1(20.0)$ & $38566528.6(0.00,-)$ \\
Oral\&/or esophageal thrush & $4(80.0)$ & $0(0)$ & 1 \\
Others & $2(100.0)$ & $69(28.5)$ & \\
Missing & $173(71.5)$ & & \\
\hline
\end{tabular}

$* *$ p-value $<0.001$

Note: Adjusted for sex, age group, income, ART regimen, clinical stage of disease and educational status were not done because they were insignificant for bivariate analysis. 


\subsection{Discussion}

Malnutrition and HIV/AIDS effects are interrelated and exacerbate one another in a vicious cycle. Both HIV/AIDS and malnutrition independently cause progressive damage to the immune system and increased susceptibility to infection. Since the introduction of ART, the prevalence of malnutrition has diminished but not yet disappeared. The prevalence of malnutrition varied based on client's ART duration.

\subsubsection{Socio Demographic Characteristics}

Initially, this study was conducted to assess the nutritional status of APLWHIV that are on ART and factors associated. This quantitative cross-sectional study in which two health facilities involved indicated that clients malnourished were $26.47 \%$ and $73.53 \%$ were well nourished. Females accounted $65.5 \%$, of them $29.2 \%$ were malnourished. Males accounted $134.5 \%$, of them $24.1 \%$ were malnourished. This proportion of malnutrition was much higher in females who were on ART. Similar study done in Bahir Dar showed proportion of malnourished females were $46 \%$ less likely to be malnourished than males, $\mathrm{AOR}=0.54,95 \% \mathrm{CL}(0.52$ 0.95) which is opposite to this study. This may be due to females do not give attention for themselves. In order to sustain their children and husbands life they give attention for their family. As we can see above, to become male is protective to be malnourished as it is shown by logistic regression analysis. That means females are more vulnerable to the problem. Therefore, there is significant difference between male and female.

Food insecurity occurs more often in PLHIV as the majority of HIV infections occur in the most productive section of the population, which is amongst adults aged 1549 (FAO 2003). This may strengthen the result showed in this study. From the respondents that are malnourished (112), adults aged $15-29$ account $(24.26 \%), 30-49=(27.6 \%)$ and $>=50=(20.0 \%)$, respectively. The study shows that $28.3 \%$ of the respondents were divorced while $27.9 \%$, $25.0 \%, 23.5 \%$ were married, widowed and single respectively.

Majority of the respondents attained university (36.4\%) were malnourished highly and those who could read and write only were secondly affected(33.3\%).The respondents attained college, secondary, elementary and preparatory were $(30.0 \%),(25.6 \%),(25.4 \%)$ and $(21.4 \%)$ respectively. Results from placebo-controlled trial in Dar es Salaam, Tanzania, found that the woman's age, education level, and height were inversely related to the incidence of wasting.

The study shows that $(26.6 \%)$ of the respondents that got $<$ 675ETB monthly income were malnourished and $(26.3 \%)$ of the respondents that are malnourished were got $>_{=675 \mathrm{ETB}}$ monthly income. This study is not comparable with the idea of Black, R.E. etal. 2008) which showed Income, poverty (due to unemployment, low wages, or lack of education) can lead to household food insecurity, inadequate care, "unhealthy household environment, and lack of health services. People of low socioeconomic status are most vulnerable to food insecurity since purchasing power serves as a main determinant of the ability-to-afford nutritional food sources. The probable reason may be; presence of opportunistic infection in both, lack of dietary counseling and the difference of study period and difference in the study population. In addition Hospital based study in 1998 in Spain found out the patient's socioeconomic situation is not influenced by nutritional condition except for the work, affectivity and family environment (Sanchez etal. (1998).

\subsubsection{Associated Factors of Nutritional Status}

The findings of this study indicated that, stage of HIV didn't show any significant effect on respondent's nutritional status. However, $(30 \%)$ of respondents that are malnourished were on stage I, $(20.4 \%)$ are on stage II, $(27.6 \%)$ are on stage III and stage IV $(0 \%)$. There were no respondents that are malnourished on stage four which is not consistent with other studies. This may be due to low number of respondents $2(0.4 \%)$ that are on stage four. According to study in Oslo, Norway in 1993, patients with HIV-infection often shows progressive loss of weight and poor nutritional status, which may appear during all stages of the HIV-infection (Thommessen, M. and Rund berget J. 1993).

According to the finding of this research, there was no statistically significant difference in between respondents getting nutritional care and support and those not getting care and support with their nutritional status. But the study showed that $28.7 \%$ of the respondents were malnourished from $(63.1 \%)$ that are not getting any nutritional care and support. From those getting support (36.9\%), (22.9\%) of them was malnourished. This is opposite with study in Zambia found that Interventions using supplements or food at time of ART commencement may improve treatment outcomes and providing food support to food insecure patients increased ART adherence by $40 \%$ and increased weight during the first 6 months of treatment (Megazzini KS. et al. 2006). This may be due to inadequate nutritional support, delay in care and support given for the malnourished clients.

Tuberculosis (TB) is the most common opportunistic infection among individuals with HIV/AIDS. From the written materials, HIV-related infections, such as tuberculosis and diarrhea, not only have nutritional status as a significant determinant of their incidence and severity, but they also have severe nutritional consequences that commonly precipitate appetite loss, weight loss and wasting (WHO 2003). This study also showed (75.9\%) of the malnourished respondents had tuberculosis. But the idea of WHO could not be seen in line with other opportunistic infection that didn't show any significant effect on the nutritional status of the respondents. This may be due to early treatment of the diseases. This will need further investigation by future researchers in the specified area to clearly explore why other opportunistic infections didn't show statistically significant effect on the nutritional status of APLWHIV that are on ART since they happen due to side effect of 
medication. When we compare the percent of the effect of opportunistic infections of respondents on their nutritional status, those with chronic diarrhea that are malnourished are $20.4 \%$, with chronic cough were $19.6 \%$, oral and esophageal problems $20.0 \%$ and others account $28.5 \%$.

In this study about $(39.0 \%)$ of the respondents were malnourished from those interrupted ART treatment. But from those not interrupted treatment, (24.5\%) were malnourished. This shows interrupting treatment had a significant effect on the nutritional status of the study subject. This study is comparable with different studies done. In Some PLHIV, starting antiretroviral therapy may result in increased appetite (Godfrey and Xuereb, 2004)

\subsubsection{Nutritional Statuses of the Respondents}

In this study the prevalence of malnutrition in HIV/AIDS clients who attend ART was (27\%). The prevalence was much lower than a study done in India (72\%) (SatiB, etal. (2004), Malawi (57\%) (R.Zacharia etal. (2002) and greater than study done in Boston (18\%) (OlalekanA and Uthmen 2008), Bahir Dar (21.1\%) (MollaD. , Fekadu M. and Dereje B. (2013) and in Addis Ababa (18\%) (MOH \&HAPCO 2008) and SSA (10.3\%) (Mengeli,A. etal. (2006), Lusaka Zambia (15.8\%) (Kelly,P. etal. (1996). The difference may be due to, socio culture, year of study and awareness creation made regarding HIV and nutrition.

\section{Conclusion}

The study shows the presence of malnutrition and associated risk factors in Nekemte Hospital and Nekemte health center. About 112 respondents were malnourished. From the associated factors tuberculosis played a great role. In Nekemte Referral Hospital and Nekemte Health center clients are malnourished due to the associated factors such as presence of opportunistic infection that is tuberculosis. From 19 clients that have TB (75.9\%) of them are mal nourished. Interrupting treatment was seen to contribute to malnutrition for the respondents. From the socio demographic factors the respondent's age, marital status, income and educational status didn't show significances on the respondents' nutritional status in both Bivariate and multivariate analysis. In this study some associated factors of nutritional status such as ART regimen, clinical stage of the disease and nutritional care and support, didn't show any significant effect on clients' nutritional status.

\section{References}

[1] Aluísio J D Barros and Cesar G Victora. A nationwide wealth score based on the 2000 Brazilian demographic census. Rev SAude Publica 2005; 39(4).

[2] Amadi B, Kelly P, Mwiya M, Mulwazi E, Sianongo S, Changwe F, et al. Intestinal and systemic infection, HIV, and Journal of Postgraduate Medicine L. M. Houtzager 72 mortality in Zambian children with persistent diarrhoea and malnutrition. J Pediatr Gastroenterol Nutr 2001; 32(5):550-4.
[3] CSA and ORC Macro. 2006. Ethiopia Demographic and Health Survey 2005. Addis Ababa, Ethiopia and Calverton, Maryland, USA: CSA and ORC Macro.

[4] CSA. Ethiopian Demographic and Health Survey; 2005.

[5] Disease Prevention and Control Department - Federal Ministry of Health, Ethiopia. Aids inEthiopia:5thReport,2004.

[6] FAO of United Nations. Health and agricultural report. 2000

[7] Godfrey C. Xuereb. Healthy Eating for Better Living: A Manual on Nutrition and HIV/AIDS for Healthcare Workers in the Caribbean. Vol. 37, No.4, 2004.

[8] Judith Nerad, et al. General Nutrition Management in HIV • Clinical Infectious Diseases 2003:36 (Suppl 2) : S53.

[9] Kelly, P.; Summerbell, C.; Ngwenya, B.; Mandanda, B.; Hosp, M.; Fuchs, D.; Wachter, H.; Luo, N. P.; Pobee, J. O. M.; Farthing, M. J. G. Systemic immune activation as a potential determinant of wasting in Zambians with HIV-related diarrhea. Qjm. 89(11):831-837, November 199625.

[10] Mangili A, Murman DH, Zampini AM, Wanke CA. Nutrition and HIV infection: review of weight loss and wasting in the era of highly active antiretroviral therapy from the nutrition for healthy living cohort. Journal of Clinical Infectious Diseases. 2006 Mar 15; 42(6): 836-42.

[11] Megazzini KS, Washington M, Sinkala M, Lawson-Marriot S, Stringer E, Krebs D, et al . A pilot randomised trial of nutritional supplements in food insecure patients receiving antiretroviral therapy in Zambia. In: Sixteenth International AIDS Conference; 2006; Toronto, Canada; 2006.

[12] MOH and HAPCO. 2008. Effectiveness of ART Program in Ethiopia. Addis Ababa: unpublished.

[13] MOH. 2006. AIDS in Ethiopia 6th Report. Addis Ababa: $\mathrm{MOH} / \mathrm{HAPCO}$.

[14] MOH. 2008b. National Nutrition and HIV/AIDS Implementation Reference Manual. Addis Ababa: $\mathrm{MOH}$.

[15] Molla Daniel, Fekadu Mazengia, Dereje Birhanu. Nutritional Status and Associated Factors Among Adult HIV/AIDS Clients in Felege Hiwot Referral Hospital, Bahir Dar, Ethiopia. Science Journal of Public Health. Vol. 1, No. 1, 2013, pp. 24-31.

[16] Olalekan A Uthman. Prevalence and pattern of HIV-related malnutrition among women in sub-Saharan Africa: metaanalysis of demographic health surveys, July, 2008.

[17] R. Zacharia, M. P. Spiemann, A. D. Harries and F. M. L. salanponi moderate to severe malnutrition in a patient with tuberculosis is a risk factor associated with early death; National Tuberculosis control program of Malawi community health science unit private; 2002, page 65 .

[18] Raiten D, Grinspoon S, Arpadi S. Nutritional considerations in the use of ART in resourcelimited settings. Geneva: World Health Organization; 2005.

[19] Reniers, G., T. Araya, and G. Davey. 2009. - Steep Decline in Population-level AIDS Mortality Following the Introduction of Antiretroviral Therapy in Addis Ababa, Ethiopia.l AIDS 23: 511-518

[20] Reynolds SJ et al. Antiretroviral therapy where resources are limited. New England Journal of Medicine, 2003, 348:1806-1809 
[21] Sanchez Alvarez MC, et al. Evolution of the nutritional status of patients with HIV-AIDS. Effects of socioeconomic situation and dietetic counseling. Annale de Medicina Interna. 1998 Dec;15(12):627-32.

[22] Sati B, Garg DK, Purohit SD, Rathore R, Haag A, Mora C; Prevalence of malnutrition among HIV infected individualsin Rajasthan, India. Jul: 2004 abstract no. MoPeB3267.

[23] Thanker, H. K., and M. H. Snow. "HIV Viral Suppression in the Era of Antiretroviral Therapy." Postgraduate Medical Journal (January 2003): 36.

[24] Thommessen M, Rundberget J. Nutritional counseling to patients with HIV infection. Can nutritional intervention prevent, expose or relieve symptoms in HIV-positive persons?
Tidsskr Norwegian Laegeforen. 1993 Jan 30; 113(3):324-6.

[25] Tony Castleman, Eleonore Seumo-Fosso, and Bruce Cogill. Food and Nutrition Implications of Antiretroviral Therapy in Resource Limited Settings. Report on HIV/AIDS. May 2004.

[26] WHO, Interim WHO Clinical Staging Of Hiv/Aids And Hiv/Aids Case Definitions For Surveillance, African Region. World Health Organization 2005.

[27] WHO, Nutrient requirements for people living with HIV/AIDS : report of a technical consultation, World Health Organization, Geneva, 13-15 May 2003.

[28] World Health Organization (WHO). Treat 3 million by 2005 ("3 by 5 "). 